\title{
PESQUISAS QUALITATIVAS EM ENGENHARIA E GESTÃO DO CONHECIMENTO: UMA REVISÃO SISTEMÁTICA
}

\author{
INVESTIGACIÓN CUALITATIVA EN INGENIERÍA Y GESTIÓN \\ DEL CONOCIMIENTO: UNA REVISIÓN DE LA LITERATURA
}

\begin{abstract}
Paloma Maria Santos - paloma@egc.ufsc.br Doutora em Engenharia e Gestão do Conhecimento pela Universidade Federal de Santa Catarina (UFSC).

Airton Zancanaro - airtonz@egc.ufsc.br Doutor em Engenharia e Gestão do Conhecimento pela Universidade

Federal de Santa Catarina (UFSC).
\end{abstract}

Marina Keiko Nakayama - marina@egc.ufsc.br Doutora em Administração na Universidade Federal do Rio Grande do Sul (UFRGS).

RESUMO

Introdução: Na Economia do Conhecimento as demandas organizacionais surgem cada vez mais complexas, passando a exigir sistemas com desempenho de tarefas intensivas em conhecimento. Neste contexto, encontra-se a Engenharia e Gestão do Conhecimento cujo foco de estudos é a pesquisa, concepção, desenvolvimento e aplicação de sistemas de apoio a tarefas intensivas em conhecimento.

Objetivo: Este artigo objetiva investigar a ocorrência de trabalhos que exploram o tema pesquisa qualitativa na área da Engenharia e Gestão do Conhecimento por meio de uma revisão sistemática.

Metodologia: Utilizou-se a base de dados SCOPUS, de forma a localizar estudos sobre o tema no período de 2006 a 2011.

Resultados: Pôde-se constatar a carência e a insipiência de publicações de pesquisas sobre o tema, o que sugere a oportunidade e um relativo espaço para pesquisas futuras.

Conclusões: $\mathrm{O}$ uso deste tipo de pesquisa por outros pesquisadores facilita e potencializa o avanço e desenvolvimento da ciência. 
Palavras-chave: Revisão sistemática. Pesquisa qualitativa. Engenharia do conhecimento. Gestão do conhecimento.

\section{INTRODUÇÃO}

O conhecimento é tido como de utilidade, sendo um meio para obter resultados econômicos e sociais. Ele dá a habilidade para o indivíduo agir de forma a guiar as suas ações de maneira apropriada, por meio da correta utilização das informações, sendo este, o objeto de estudo da área da Engenharia e Gestão do Conhecimento (EGC).

A Gestão do Conhecimento tem como objetivo a definição das questões relacionadas à estrutura organizacional, envolvendo pessoas e processos. Já Engenharia, cuida dos aspectos técnicos e metodologias que facilitam os processos de elucidação e propõem soluções às tarefas de interesse estratégico da Gestão.

Uma revisão de literatura sobre aplicação do método qualitativo na área da EGC pode ser de grande valia para os pesquisadores, na medida em que estes, por vezes, se deparam com a dificuldade em selecionar trabalhos científicos adequados a realidade de sua investigação. Trabalhos desta natureza também podem ser úteis para o aprofundamento de uma pesquisa ou mesmo para a identificação de temas emergentes para estudo.

Dessa forma, o presente artigo objetiva investigar a ocorrência de trabalhos que exploram o tema pesquisa qualitativa na área da EGC por meio de uma revisão sistemática. Utilizou-se a base de dados SCOPUS de forma a localizar estudos sobre o tema no período de 2006 a 2011.

Na seção 2 apresenta-se uma revisão sobre a EGC bem como sobre método qualitativo na seção 3. A seção 4 aborda o procedimento metodológico utilizado para a elaboração desta pesquisa. Na seção 5 tem-se os resultados e, finalmente, a seção 6 traz as considerações finais do artigo e as oportunidades de trabalhos futuros. 


\section{ENGENHARIA E GESTÃO DO CONHECIMENTO}

A compreensão da era em que vivemos apóia-se cada dia mais sobre a chamada sociedade em rede. Fortemente impactante nas últimas duas décadas, a sociedade em rede traz como principal elemento a modificação do âmbito social, econômico e tecnológico. Os tradicionais fatores de produção - terra, trabalho e capital - não desapareceram, mas tornaram-se secundários. Eles podem ser obtidos, e obtidos facilmente, desde que exista conhecimento.

Neste novo cenário, o conhecimento é tido como uma utilidade, como um meio de obter resultados econômicos e sociais (DRUCKER, 1993). O que caracteriza o atual contexto histórico, marcado pela revolução tecnológica, não é a centralidade da informação e do conhecimento, mas a aplicação desses para a geração de novos conhecimentos (CASTELLS, 1999).

O conhecimento envolve a capacidade contínua de agir (SVEIBY, 1998) e é o único recurso significativo atualmente (DRUCKER, 1993). Ele dá à pessoa habilidade de uso da informação para guiar as ações de maneira apropriada à situação (KALPIC; BERNUS, 2006), permitindo gerar um diferencial nos resultados das organizações e nos seus meios produtivo e comercial. Esse diferencial de valoração do produto proporciona a sua aceitação no mercado, caracterizando a Economia do Conhecimento.

Nesta Economia, as demandas organizacionais surgem cada vez mais complexas, passando a exigir sistemas com desempenho de tarefas intensivas em conhecimento. Neste contexto é que se insere Engenharia e Gestão do Conhecimento, cujo foco de estudo está centrado na pesquisa, concepção, desenvolvimento e aplicação de sistemas para apoio ao ciclo de atividades e macroprocessos intensivos em conhecimento, entendido como fator gerador de valor.

Enquanto a Gestão do Conhecimento define as questões da estrutura organizacional, que envolvem pessoas e processos, a Engenharia do Conhecimento cuida dos aspectos técnicos e metodologias que facilitam o processo de elucidar e propor soluções às tarefas de interesse estratégico da Gestão do Conhecimento (BERZTISS, 2006).

Nesse âmbito de estudos, o conhecimento permeia as pesquisas de diversas maneiras, se apresentando como objeto de acordo com seus processos relacionados no ciclo de desenvolvimento, quais sejam: criação, aquisição, 
compartilhamento, disseminação, elicitação, codificação, armazenamento, transferência, utilização, aprimoramento e integração.

\section{PESQUISA QUALITATIVA}

No final do século XIX, em Heidelberg, Alemanha e no início do século XX, em Chicago, Estados Unidos, nascia uma escola de sociologia que se rebelava radicalmente contra o positivismo, no qual, assumia como verdade apenas o que cabia no método ao invés de privilegiar a captação da realidade humana (MINAYO; SANCHES, 1983; DEMO, 1998).

Para Minayo e Sanches (1983), o sociólogo alemão Max Weber foi o que deu uma "maior consistência metodológica" (p.243) a escola de sociologia, afirmando que é de responsabilidade das ciências sociais compreender as ações humanas e não apenas reduzir a descrição dos comportamentos. Com o tempo e as discussões em torno da fenomenologia e da hermenêutica aumentaram e o método qualitativo começou a ser evidenciado.

Segundo Demo (1998), inicialmente, o método qualitativo não tinha nada em comum em relação a outros métodos a não ser a oposição ao quantitativo. Com o passar do tempo as técnicas foram sendo aperfeiçoadas focando nos problemas humanos e sociais (MINAYO; SANCHES, 1983).

Atualmente a pesquisa qualitativa trabalha num universo de "[...] valores, crenças, hábitos, atitudes, representações, opiniões [...]" (PAULILO, 1999, p.135), específicos de indivíduos ou grupos, sendo portando humanista. Taylor e Bogdan (1987), afirmam que o método qualitativo é uma arte, produzindo investigações indutivas e dados descritivos. Todas as perspectivas (cenários e pessoas) são valiosas e dignas de estudo. Deste modo, o investigador percebe o cenário e as pessoas com uma visão holística, separando as suas próprias crenças, perspectivas ou predisposições.

A pesquisa qualitativa trabalha essencialmente com dois tipos de dados: os dados verbais que são coletados em entrevistas semi-estruturadas ou como narrativas e os dados visuais como a observação participante e não-participante à etnografia (FLICK, 2004). Portanto, ela é utilizada para a compreensão de fenômenos que podem ser caracterizados por um alto grau de complexidade 
(PAULILO, 1999), preocupando-se com aspectos da realidade que não podem ser quantificados (FONSECA, 2002).

\section{PROCEDIMENTOS METODOLÓGICOS}

Este artigo utiliza o método da revisão sistemática para investigar a ocorrência de estudos sobre pesquisa qualitativa na área da Engenharia e Gestão do Conhecimento.

A revisão sistemática é um tipo de delineamento de pesquisa, amplamente utilizado pelas ciências médicas, que tem como principal característica o fornecimento de evidências bases para a tomada de decisão, sendo que sua aplicação pode ser estendida para outras áreas de conhecimento.

Trata-se de um método de pesquisa que utiliza como fonte de dados a literatura sobre determinado tema. Esse tipo de investigação disponibiliza um resumo das evidências relacionadas a uma estratégia de intervenção específica, mediante a aplicação de métodos sistemáticos de busca para apreciação crítica e síntese da informação selecionada. As revisões sistemáticas podem apresentar resultados conflitantes e/ou coincidentes, bem como identificar temas que necessitam de evidência, auxiliando na orientação para investigações futuras (UNIVERSITY OF YORK, 2001; SAMPAIO; MANCINI, 2007).

As revisões sistemáticas são planejadas para ser metódicas, explícitas e passíveis de reprodução. Elas partem de uma pergunta de pesquisa clara, da definição de uma estratégia de busca, do estabelecimento de critérios de inclusão e exclusão dos artigos e, acima de tudo, de uma análise criteriosa da qualidade da literatura selecionada, conforme apresentado na Figura 1 (SAMPAIO; MANCINI, 2007).

Com base nisso, este artigo partiu da seguinte pergunta de pesquisa: Quais trabalhos têm explorado o tema: Pesquisa qualitativa na área de Engenharia e Gestão do Conhecimento?

De acordo com as instruções de Sampaio e Mancini (2007), definiu-se as estratégias de busca, bem como, os critérios de inclusão e exclusão dos artigos.

As estratégias de busca resumem-se a: 
- Realização de uma busca utilizando os termos específicos "management" E "engineering" E "knowledge" E "qualitative" na base de dados SCOPUS ${ }^{1}$ (em 20/09/2011);

- Seleção dos seguintes tipos de trabalhos: "article", "review", "conference paper" e "conferece review";

- Inclusão dos trabalhos publicados entre os anos de 2006-2011;

- Exportação dos dados dos trabalhos resultantes da busca para uma planilha Excel.

Tais critérios de inclusão envolvem:

- Conter no título do trabalho, no resumo ou nas palavras-chave os termos: "management" E "engineering" E "knowledge" E "qualitative";

- Serão incluídos trabalhos que efetivamente respondam a pergunta de pesquisa.

${ }^{1}$ A SCOPUS é uma base de dados multidisciplinar que é mantida pela Editora Elsevier desde 2004. Seu endereço eletrônico é http://www.scopus.com/ 
Figura 1 - Procedimentos metodológicos para realização de uma revisão sistemática.

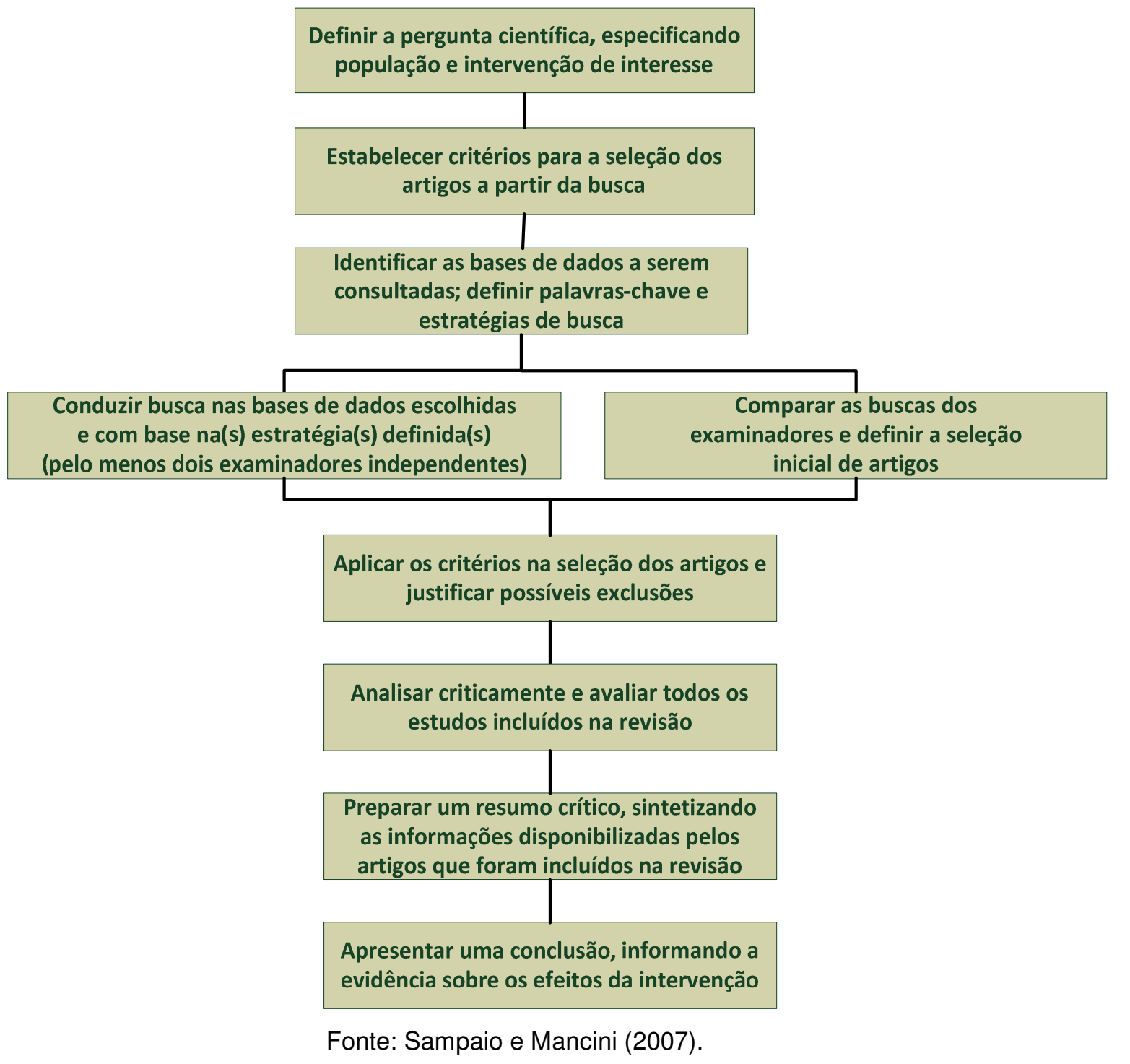

A Figura 2 abaixo representa como se deu a aplicação dos procedimentos metodológicos aqui descritos. 
Figura 2 - Representação da aplicação dos procedimentos metodológicos aplicados neste artigo.

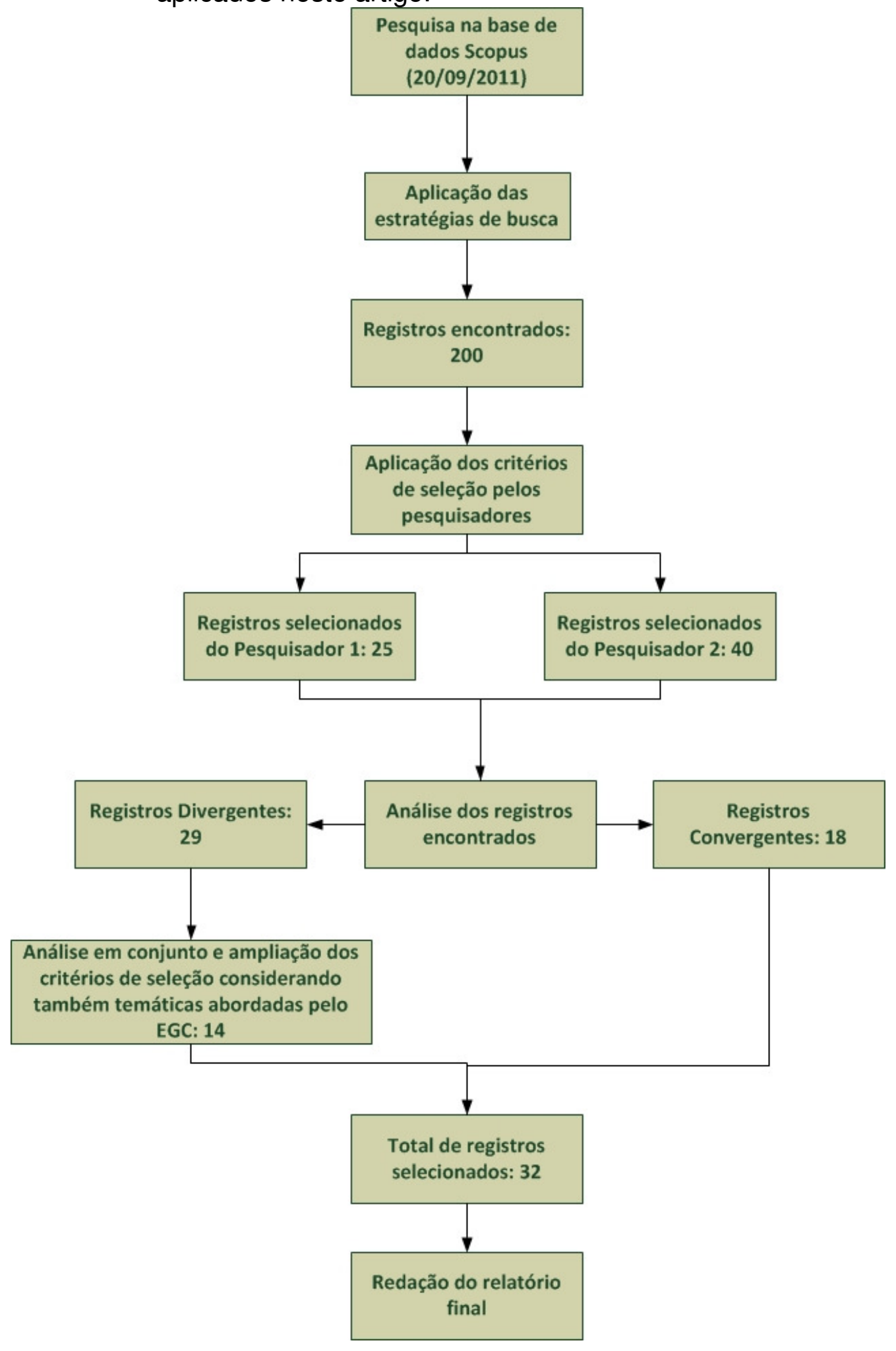

Fonte: Elaborado pelos autores.

Os resultados desses procedimentos metodológicos são apresentados na próxima seção deste artigo. 


\section{RESULTADOS DA PESQUISA}

Após a aplicação das estratégias de busca, a base de dados SCOPUS retornou 200 trabalhos, conforme Gráfico 1 abaixo.

Gráfico 1 - Registros encontrados após aplicação das estratégias de busca.

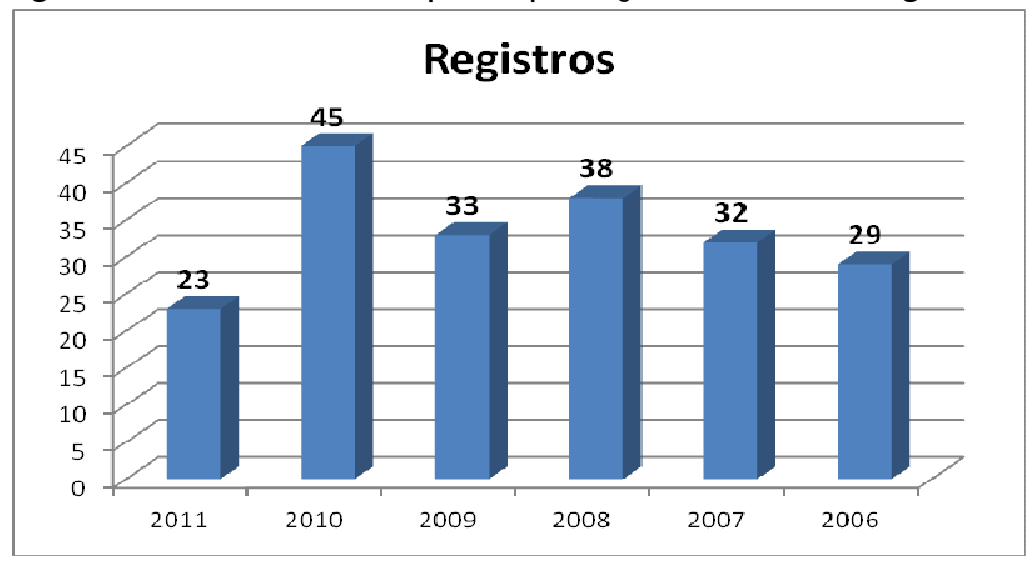

Fonte: Elaborado pelos autores.

Destes 200 trabalhos, a grande maioria estava classificado ou como Conference Paper ou como Article, conforme Quadro 1.

Quadro 1 - Classificação dos documentos encontrados após aplicação das estratégias de busca.

\begin{tabular}{|l|c|}
\hline \multicolumn{1}{|c|}{ Tipos de Documento } & Quantidade \\
\hline Conference Paper & 100 \\
\hline Article & 92 \\
\hline Review & 5 \\
\hline Conference Review & 3 \\
\hline
\end{tabular}

Fonte: Elaborado pelos autores.

Percebe-se uma grande incidência de trabalhos da sub área de conhecimento Computer Science e Engineering, com respectivamente 94 e 70 trabalhos. A incidência das demais áreas está demonstrada no Quadro 2, abaixo. 
Quadro 2 - Incidência de trabalhos de acordo com a sub área de conhecimento

\begin{tabular}{|l|c|}
\hline \multicolumn{1}{|c|}{ Sub Área } & Quantidade \\
\hline Computer Science & 94 \\
\hline Engineering & 70 \\
\hline Mathematics & 35 \\
\hline Business, Management and Accounting & 35 \\
\hline Social Sciences & 25 \\
\hline Decision Sciences & 20 \\
\hline Medicine & 14 \\
\hline Environmental Science & 13 \\
\hline Biochemistry, Genetics and Molecular & 9 \\
Biology & \\
\hline Physics and Astronomy & 5 \\
\hline Energy & 4 \\
\hline Earth and Planetary Sciences & 4 \\
\hline Chemical Engineering & 4 \\
\hline Economics, Econometrics and Finance & 3 \\
\hline Health Professions & 2 \\
\hline Materials Science & 2 \\
\hline Psychology & 2 \\
\hline Agricultural and Biological Sciences & 1 \\
\hline Arts and Humanities & 1 \\
\hline Multidisciplinary & 1 \\
\hline Neuroscience & 1 \\
\hline Pharmacology, Toxicology and & 1 \\
\hline Pharmaceutics & \\
\hline Fonte: Elaborado pelos autores. & \\
\hline
\end{tabular}

Fonte: Elaborado pelos autores.

Além disso, nota-se que $19 \%$ dos trabalhos possuem como País de afiliação do Autor os Estados Unidos. O Brasil aparece com 2\%, juntamente a Suiça, Finlândia, França, Turquia e Japão, conforme Quadro 3. 
Quadro 3 - Incidência de trabalhos de acordo com o País de Afiliação do Autor.

\begin{tabular}{|l|c|}
\hline \multicolumn{1}{|c|}{ País } & Quantidade \\
\hline United States & 38 \\
\hline United Kingdom & 18 \\
\hline Canada & 14 \\
\hline China & 12 \\
\hline Germany & 11 \\
\hline Sweden & 8 \\
\hline Austria & 8 \\
\hline Australia & 8 \\
\hline Netherlands & 7 \\
\hline Spain & 6 \\
\hline Denmark & 5 \\
\hline Italy & 5 \\
\hline Brazil & 4 \\
\hline Switzerland & 4 \\
\hline Finland & 4 \\
\hline France & 4 \\
\hline Turkey & 4 \\
\hline Japan & 4 \\
\hline Malaysia & 3 \\
\hline South Africa & 3 \\
\hline Ireland & 3 \\
\hline New Zealand & 3 \\
\hline India & 3 \\
\hline Norway & 3 \\
\hline Saudi Arabia & 3 \\
\hline Portugal & 2 \\
\hline Israel & 2 \\
\hline Iran & 2 \\
\hline Russian Federation & 2 \\
\hline Belgium & 2 \\
\hline Singapore & 1 \\
\hline Taiwan & 1 \\
\hline Thailand & 1 \\
\hline Uzbekistan & 1 \\
\hline Viet Nam & 1 \\
\hline Serbia & 1 \\
\hline Romania & 1 \\
\hline Nigeria & \\
\hline Mexico & \\
\hline Lithuania & \\
\hline Fonte: Elaboradopes & 1 \\
\hline
\end{tabular}

Fonte: Elaborado pelos autores.

Tendo em vista verificar se os trabalhos atendiam aos critérios de inclusão estabelecidos, procedeu-se com a leitura dos títulos, palavras-chave indexadas pelo sistema e pelos autores e os resumos (abstracts) dos trabalhos. Cada uma dos pesquisadores, em separado, fez esta avaliação inicial. O pesquisador 1 selecionou 25 trabalhos, enquanto que o pesquisador 2 selecionou 40. De todos os trabalhos selecionados, 18 eram convergentes, ou seja, foram selecionados por ambos os pesquisadores. Em uma reunião de consenso, buscou-se avaliar as divergências 
encontradas nos 29 trabalhos restantes. Tal representação está sintetizada na Figura 2, demonstrada acima.

Tendo em vista a incidência desses 29 trabalhos que traziam implicitamente os termos "Engenharia" e "Gestão" do Conhecimento numa abordagem de pesquisa qualitativa, decidiu-se ampliar os critérios de inclusão considerando o grau de pertinência desses trabalhos a esta pesquisa. Dessa forma, passou-se a aceitar também trabalhos que viessem a relacionar as temáticas afins abordadas pelas áreas em estudo, conforme Figura 3.

Figura 3 - Representação das temáticas afins consideradas na ampliação dos critérios de inclusão.

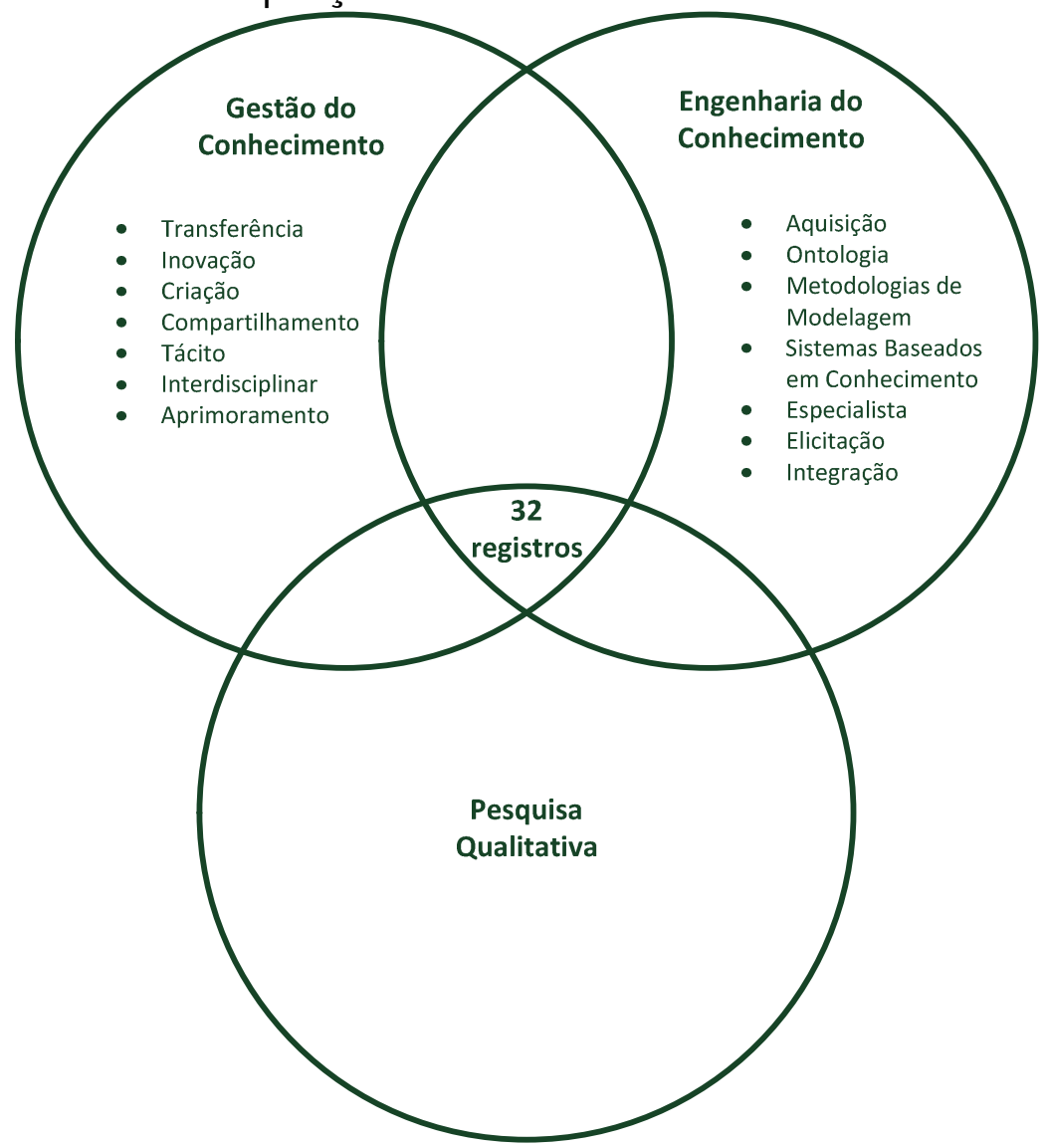

Fonte: Elaborado pelos autores.

Foram recuperados através deste processo 14 trabalhos. Somados aos 18 convergentes, resultantes da primeira avaliação, totalizaram 32 registros. Tais trabalhos foram categorizados conforme a incidência dos termos "Engenharia e Gestão do Conhecimento E Pesquisa Qualitativa" (incidência explícita - Quadro 4); "Engenharia do Conhecimento E Pesquisa Qualitativa" (Quadro 5); "Gestão do Conhecimento E Pesquisa Qualitativa" (Quadro 6) e "Engenharia e Gestão do 
Paloma Maria Santos; Airton Zancanaro; Marina Keiko Nakayama

Pesquisas qualitativas em engenharia e gestão do conhecimento: uma revisão sistemática

Conhecimento E Pesquisa Qualitativa" (incidência implícita - quando se referiam as temáticas afins apresentadas na Figura 3 - Quadro 7).

Quadro 4 - Trabalhos com incidência explícita dos termos Engenharia e Gestão do Conhecimento e Pesquisa Qualitativa.

\begin{tabular}{|c|c|c|}
\hline \multicolumn{3}{|c|}{ EC+GC+Quali - Incidência explícita } \\
\hline Título & Autor(es) & Ano \\
\hline $\begin{array}{l}\text { An analysis of collaborative patterns in large- } \\
\text { scale ontology development projects }\end{array}$ & $\begin{array}{l}\text { Falconer S., Tudorache T., Noy } \\
\text { N.F. }\end{array}$ & 2011 \\
\hline $\begin{array}{l}\text { Managing the project learning paradox: A } \\
\text { set-theoretic approach toward project } \\
\text { knowledge transfer }\end{array}$ & $\begin{array}{l}\text { Bakker R.M., Cambre B., Korlaar } \\
\text { L., Raab J. }\end{array}$ & 2011 \\
\hline $\begin{array}{l}\text { Technology adoption in the BIM } \\
\text { implementation for lean architectural practice }\end{array}$ & $\begin{array}{l}\text { Arayici Y., Coates P., Koskela L., } \\
\text { Kagioglou M., Usher C., O'Reilly } \\
\text { K. }\end{array}$ & 2011 \\
\hline $\begin{array}{l}\text { Knowledge integration in global R\&D } \\
\text { networks }\end{array}$ & $\begin{array}{l}\text { Erkelens R., Van Den Hooff B., } \\
\text { Vlaar P., Huysman M. }\end{array}$ & 2010 \\
\hline $\begin{array}{l}\text { Preliminary approach to improve knowledge } \\
\text { management in engineering management }\end{array}$ & Abdul-Rahman H., Wang C. & 2010 \\
\hline $\begin{array}{l}\text { Informing UK Information management } \\
\text { pedagogic practice: The nature of } \\
\text { contemporary higher education culture }\end{array}$ & Grant K., Hackney R., Edgar D. & 2010 \\
\hline $\begin{array}{l}\text { Uncovering cultural perceptions and barriers } \\
\text { during knowledge audit }\end{array}$ & $\begin{array}{l}\text { Levy M., Hadar I., Greenspan S., } \\
\text { Hadar E. }\end{array}$ & 2010 \\
\hline $\begin{array}{l}\text { Cross-functional emerging domains in } \\
\text { engineering design: Actor-networks and } \\
\text { collaborative models, organizational learning } \\
\text { and knowledge management }\end{array}$ & Figueiredo J. & 2008 \\
\hline $\begin{array}{l}\text { Organizational learning during } \\
\text { internationalization: Acquiring local } \\
\text { institutional knowledge }\end{array}$ & Javernick-Will A.N. & 2009 \\
\hline $\begin{array}{l}\text { Intelligent support of the preprocessing } \\
\text { stage of engineering analysis using case- } \\
\text { based reasoning }\end{array}$ & $\begin{array}{l}\text { Wriggers P., Siplivaya M., } \\
\text { Joukova I., Slivin R. }\end{array}$ & 2008 \\
\hline $\begin{array}{l}\text { Organizing and managing personal } \\
\text { electronic files: A mechanical engineer's } \\
\text { perspective }\end{array}$ & $\begin{array}{l}\text { Hicks B.J., Dong A., Palmer R., } \\
\text { McAlpine H.C. }\end{array}$ & 2008 \\
\hline $\begin{array}{l}\text { Structuring cross-organizational knowledge } \\
\text { sharing }\end{array}$ & White K.F., Lutters W.G. & 2007 \\
\hline $\begin{array}{l}\text { A study on the critical success factors for } \\
\text { corporations embarking on knowledge } \\
\text { community-based e-learning }\end{array}$ & Chen R.-S., Hsiang C.-H. & 2007 \\
\hline $\begin{array}{l}\text { Factors inducing knowledge creation: } \\
\text { Empirical evidence from Thai construction } \\
\text { projects }\end{array}$ & Teerajetgul W., Charoenngam C. & 2006 \\
\hline $\begin{array}{l}\text { Towards a better understanding of process } \\
\text { documentation }\end{array}$ & Ungan M. & 2006 \\
\hline $\begin{array}{l}\text { Men and measures: Capturing knowledge } \\
\text { requirements in firms through qualitative } \\
\text { system modelling }\end{array}$ & Swart J., Powell J.H. & 2006 \\
\hline
\end{tabular}

Fonte: Elaborado pelos autores. 
Paloma Maria Santos; Airton Zancanaro; Marina Keiko Nakayama

Pesquisas qualitativas em engenharia e gestão do conhecimento: uma revisão sistemática

Quadro 5 - Trabalhos com incidência dos termos Engenharia do Conhecimento e Pesquisa Qualitativa.

\begin{tabular}{|l|l|c|}
\hline \multicolumn{2}{|c|}{ EC + Quali } \\
\hline \multicolumn{1}{|c|}{ Título } & \multicolumn{1}{|c|}{ Autor(es) } & Ano \\
\hline $\begin{array}{l}\text { Enriching city entities in the EKOSS failure } \\
\text { cases knowledge base with linked open data }\end{array}$ & Guo W., Kraines S.B. & 2010 \\
\hline $\begin{array}{l}\text { Knowledge management in renewing } \\
\text { software development processes }\end{array}$ & $\begin{array}{l}\text { Kukko M., Helander N., Virtanen } \\
\text { P. }\end{array}$ & 2008 \\
\hline
\end{tabular}

Fonte: Elaborado pelos autores.

Quadro 6 - Trabalhos com incidência dos termos Gestão do Conhecimento e Pesquisa Qualitativa.

\begin{tabular}{|l|l|c|}
\hline \multicolumn{2}{|c|}{ Titulo } & \multicolumn{1}{|c|}{ Autor(es) } \\
\hline $\begin{array}{l}\text { Participation in intra-firm communities of } \\
\text { practice: A case study from the automotive } \\
\text { industry }\end{array}$ & Wolf P., Spath S., Haefliger S. & 2011 \\
\hline $\begin{array}{l}\text { The difficult bridge between university and } \\
\text { industry: A case study in computer science } \\
\text { teaching }\end{array}$ & Schilling J., Klamma R. \\
\hline $\begin{array}{l}\text { The nature and role of experiential } \\
\text { knowledge for environmental conservation }\end{array}$ & $\begin{array}{l}\text { Fazey I., Fazey J.A., Salisbury } \\
\text { J.G., Lindenmayer D.B., Dovers } \\
\text { S. }\end{array}$ & 2010 \\
\hline $\begin{array}{l}\text { The influence of cultural values on } \\
\text { knowledge sharing across organizational } \\
\text { boundaries }\end{array}$ & Mueller J. \\
\hline $\begin{array}{l}\text { Emerging contractors in South Africa: } \\
\text { Interactions and learning }\end{array}$ & Martin L., Root D. \\
\hline $\begin{array}{l}\text { A knowledge management process in } \\
\text { communities of practice of engineering } \\
\text { based on the SECl model for knowledge }\end{array}$ & Sarirete A., Chikh A. & 2006 \\
\hline $\begin{array}{l}\text { Workplace learning: The roles of knowledge } \\
\text { accessibility and management }\end{array}$ & $\begin{array}{l}\text { Li J., Brake G., Champion A., } \\
\text { Fuller T., Gabel S., Hatcher- } \\
\text { Busch L. }\end{array}$ & 2010 \\
\hline $\begin{array}{l}\text { The perception of production engineering } \\
\text { master degree students about the existence } \\
\text { of creation environment of knowledge }\end{array}$ & $\begin{array}{l}\text { Holanda L.M.C., de Francisco } \\
\text { A.C., Kovaleski J.L. }\end{array}$ & 2010 \\
\hline $\begin{array}{l}\text { Once upon a time in Africa: A case study of } \\
\text { storytelling for knowledge sharing }\end{array}$ & Tobin P.K.J., Snyman R. \\
\hline Fonte: Elaborado pelos autores. & 2009 \\
\hline
\end{tabular}

Fonte: Elaborado pelos autores. 
Paloma Maria Santos; Airton Zancanaro; Marina Keiko Nakayama

Pesquisas qualitativas em engenharia e gestão do conhecimento: uma revisão sistemática

Quadro 7 - Trabalhos com incidência implícita dos termos Engenharia e Gestão do Conhecimento E Pesquisa Qualitativa.

\begin{tabular}{|l|l|c|}
\hline \multicolumn{2}{|c|}{ EGC + Temas (Incidência implícita) } & \multicolumn{1}{|c|}{ Autor(es) } \\
\hline $\begin{array}{l}\text { Qualitative analysis of semantically enabled } \\
\text { knowledge management systems in agile software } \\
\text { engineering }\end{array}$ & Rech J., Bogner C. \\
\hline $\begin{array}{l}\text { Fuzzy evaluation model of accounting firm } \\
\text { knowledge management performance }\end{array}$ & Zhu Z.-H., Xue D.-W. & 2010 \\
\hline $\begin{array}{l}\text { Investigating knowledge management practices in } \\
\text { software development organizations - An Australian } \\
\text { experience }\end{array}$ & $\begin{array}{l}\text { Aurum A., Daneshgar F., Ward } \\
\text { J. }\end{array}$ & 2008 \\
\hline $\begin{array}{l}\text { Observing software testing practice from the } \\
\text { viewpoint of organizations and knowledge } \\
\text { management }\end{array}$ & $\begin{array}{l}\text { Taipale O., Karhu K., } \\
\text { Smolander K. }\end{array}$ & 2007 \\
\hline $\begin{array}{l}\text { Human-centered meta-synthetic engineering for } \\
\text { knowledge creative system }\end{array}$ & $\begin{array}{l}\text { Xia C., Ruwei D., Yaodong L., } \\
\text { Mingchang Z. }\end{array}$ & 2007 \\
\hline
\end{tabular}

Fonte: Elaborado pelos autores.

De todos os artigos selecionados, os 5 que possuem maior número de citações estão referenciados abaixo no Quadro 8.

Quadro 8 - Relação dos 5 artigos mais citados.

\begin{tabular}{|c|c|c|c|c|}
\hline Citações & Título & Autor(es) & Ano & $\begin{array}{c}\text { Tipo de } \\
\text { Documento }\end{array}$ \\
\hline 24 & $\begin{array}{l}\text { The nature and role of } \\
\text { experiential knowledge for } \\
\text { environmental conservation }\end{array}$ & $\begin{array}{l}\text { Fazey I., Fazey J.A., } \\
\text { Salisbury J.G., Lindenmayer } \\
\text { D.B., Dovers S. }\end{array}$ & 2006 & Review \\
\hline 24 & $\begin{array}{l}\text { A study on the critical success } \\
\text { factors for corporations } \\
\text { embarking on knowledge } \\
\text { community-based e-learning }\end{array}$ & Chen R.-S., Hsiang C.-H. & 2007 & Article \\
\hline 11 & $\begin{array}{l}\text { Investigating knowledge } \\
\text { management practices in } \\
\text { software development } \\
\text { organisations - An Australian } \\
\text { experience }\end{array}$ & $\begin{array}{l}\text { Aurum A., Daneshgar F., } \\
\text { Ward J. }\end{array}$ & 2008 & Article \\
\hline 5 & $\begin{array}{l}\text { Organizing and managing } \\
\text { personal electronic files: A } \\
\text { mechanical engineer's } \\
\text { perspective }\end{array}$ & $\begin{array}{l}\text { Hicks B.J., Dong A., Palmer } \\
\text { R., McAlpine H.C. }\end{array}$ & 2008 & Article \\
\hline 3 & $\begin{array}{l}\text { Organizational learning during } \\
\text { internationalization: Acquiring } \\
\text { local institutional knowledge }\end{array}$ & Javernick-Will A.N. & 2009 & Article \\
\hline
\end{tabular}

Fonte: Elaborado pelos autores. 


\section{CONSIDERAÇÕES FINAIS}

Este artigo apresentou um mapeamento dos trabalhos sobre pesquisa qualitativa em Engenharia e Gestão do Conhecimento no âmbito da base de dados SCOPUS.

A análise dos dados e a síntese dos resultados encontrados possibilitaram traçar um panorama geral de como está a produção acadêmica em relação a temática abordada.

Os trabalhos publicados que relacionam a pesquisa qualitativa na área de engenharia e gestão do conhecimento tiveram início em 2006, sendo que as subáreas que mais evidenciaram tais estudos foram as de Ciências da Computação e Engenharia.

Os norte americanos lideram na ocorrência de publicações, com 19\%, seguidos dos ingleses (9\%), canadenses $(7 \%)$ e chineses (6\%). Naturalmente, o trabalho mais citado é um dos 29 publicados em 2006: The nature and role of experiential knowledge for environmental conservation, apresentando 24 citações em trabalhos de outros autores.

O Brasil aparece em $13^{\circ}$ lugar no ranking de publicações de trabalhos neste âmbito, juntamente a Suiça, Finlândia, França, Turquia e Japão, apresentando índice de ocorrência de $2 \%$.

Com base nisso, pode-se constatar a carência e a incipiência de publicações de pesquisas sobre o tema em questão, o que sugere a oportunidade e um relativo espaço para pesquisas futuras.

Por ter utilizado um procedimento metodológico baseado na revisão sistemática, este artigo possibilita que outras pesquisas sejam desenvolvidas utilizando os mesmos critérios aqui descritos. Assim, sugerem-se futuras pesquisas que utilizem outras bases de dados e também outras técnicas estatísticas.

\section{REFERÊNCIAS}

BERZTISS, Alfs T. Capability maturity. In: Encyclopedia of Knowledge Management. London: Idea Group Reference, 2006. p. 946.

CASTELLS, Manuel. A era da informação: economia, sociedade e cultura. São Paulo: Paz e Terra, 1999. (A Sociedade em Rede, v. 1). 
DEMO, Pedro. Pesquisa qualitativa: busca de equilíbrio entre forma e conteúdo. Revista Latino-Americana de Enfermagem, Ribeirão Preto, v. 6, n. 2, p. 89-104, abr. 1998.

DRUCKER, Peter. Sociedade pós-capitalista. São Paulo: Pioneira, 1993.

FLICK, Uwe. Uma introdução a pesquisa qualitativa. 2. ed. Porto Alegre: Bookman, 2004.

FONSECA, João José Saraiva da. Metodologia da pesquisa científica. Fortaleza: Universidade Estadual do Ceará, 2002.

KALPIC, Brane; BERNUS, Peter. Business process modelling through the knowledge management perspective. Journal of Knowledge Management, v. 10, n. 3, p. 40-56, 2006.

MINAYO, Maria Cecília de Souza; SANCHES, Odécio. Quantitativo-qualitativo: oposição ou complementaridade. Cadernos de Saúde Pública, Rio de Janeiro, v. 9, n. 3, p. 237-248, 1983.

UNIVERSITY OF YORK. Centre for Reviews and Dissemination. Undertaking systematic reviews of research on effectiveness: CRD's guidance for those carrying out or commissioning reviews. $2^{\text {nd }}$ ed. New York: CRD Report Number 4, 2001. Disponível em: <http://eprints.whiterose.ac.uk/1139/>. Acesso em: 20 set. 2011.

PAULILO, Maria Angela Silveira. A pesquisa qualitativa e a história de vida. Serviço Social em Revista, Londrina, v. 1, n. 2, p.135-148, jul./dez. 1999.

SAMPAIO, Rosana F.; MANCINI, Marisa C. Estudos de revisão sistemática: um guia para síntese criteriosa da evidência científica; systematic review studies: a guide for careful synthesis of the scientific evidence. Revista Brasileira de Fisioterapia, v. 11, n. 1, p. 83-89, jan./fev. 2007.

SVEIBY, Karl Erik. A nova riqueza das organizações: gerenciando e avaliando patrimônios de conhecimento. 7. ed. Rio de Janeiro: Campus, 1998.

TAYLOR, Steven J.; BOGDAN, Robert. Introducción a lós métodos culitativos de investigación: la búsqueda de significados. Barcelona: Paidós, 1987.

PRADO JUNIOR, Caio. Teoria marxista do conhecimento e método dialético materialista. Edição eletrônica Ridendo Castigat Mores, 2001. Disponível em: <http://www.ebooksbrasil.org/adobeebook/caio.pdf>.

SANTOS, Raimundo Nonato Macedo dos. Produção científica: por que medir? o que medir? Revista Digital de Biblioteconomia \& Ciência da Informação, Campinas, v. 1, n. 1, p. 22-38, jul./dez. 2003.

SOUZA, Elisabete Gonçalves. Sociedade da informação e reestruturação produtiva: crítica à dimensão utilitarista do conhecimento. Transinformação, Campinas, v. 23, n. 3, p. 219-226, 2011. 
VAISMAN, Ester; FORTES, Ronaldo Vielmi. A categoria da reprodução: Lukács, Althusser e Bourdieu \&Passeron. In: V ENCONTRO BRASILEIRO DE EDUCAÇÃO E MARXISMO: EDUCAÇÃO E EMANCIPAÇÃO HUMANA, 3, 2011.

Anais...Florianópolis: UFSC, 2011. v. 2.

WHITLEY, Richard. Cognitive and social institutionalization of scientific specialities and research áreas. In: . (Ed.). Social processes of scientific development. London: Routledge and Kegan, 1974. p. 69-95.

\title{
Title
}

Qualitative Research in Knowledge Engineering and Management: a Systematic Review

\begin{abstract}
Introduction: In the Knowledge Economy, organizational demands are becoming increasingly more complex, requiring systems that can perform knowledge-intensive tasks. The Knowledge Engineering and Management is located in this context, which focus lies on the research, design, development and implementation of systems to support knowledgeintensive task.
\end{abstract}

Objective: This article aims to investigate the occurrence of works that explore qualitative research in the Knowledge Engineering and Management field through a systematic review.

Methods: It was used the Scopus database in order to locate studies on the subject from 2006 to 2011.

Results: It was found immaturity and lack of publications on the subject, suggesting an opportunity and an open way for future research.

Conclusions: The use of this kind of research by other researchers facilitates and enhances the science advancement and development.

Keywords: Systematic review. Qualitative research. Knowledge engineering. Knowledge management.

\section{Título}

Investigación Cualitativa en Ingeniería y Gestión del Conocimiento: una revisión de la literatura

\section{Resumen}

Introducción: En la economía del conocimiento las demandas organizacionales son cada vez más complejas y requieren sistemas capaces de manejar tareas intensivas en conocimiento. En este contexto se encuentra la Ingeniería y Gestión del Conocimiento, cuyo objeto de estudio es la investigación, diseño, desarrollo e implementación de sistemas de apoyo a las tareas intensivas en conocimiento. 
Objetivo: Este artículo tiene como objetivo investigar la ocurrencia de obras que exploran el tema de la investigación cualitativa en Ingeniería y Gestión del Conocimiento a través de una revisión de la literatura.

Metodología: Se utilizó la base de datos SCOPUS, con el fin de localizar los estudios sobre el tema en el período de 2006 a 2011.

Resultados: Se encontró una inmadurez y falta de publicaciones sobre el tema, lo que sugiere la oportunidad y el espacio para futuras investigaciones.

Conclusiones: El uso de este tipo de investigación por otros investigadores facilita y mejora el avance y el desarrollo de la ciencia.

Palabras clave: Revisión de la literatura. Investigación cualitativa. Ingeniería del Conocimiento. Gestión del conocimiento.

Recebido em: 10.06.2013

Aceito em: 04.06.2015 